\title{
Incentives and implementation in allocation problems
}

\author{
with externalities *
}

\author{
María Haydée Fonseca-Mairena ${ }^{\dagger}$
}

Matteo Triossi $i^{\ddagger}$

November 21, 2021

\begin{abstract}
We study the implementation of social choice rules in environments with externalities. We prove the impossibility of implementing efficient and $\alpha$-individually rational rules in dominant strategies. We prove that the $\alpha$-core is implementable in Nash equilibrium under mild restrictions and discuss the maximality and the minimality of our results. We extend our analysis to weakly efficient rules.

Keywords: Allocation problems, Externalities, Core, Incentives, Implementation.

Economic Literature Classification Numbers: C71, C72, C78, D62, D78.
\end{abstract}

*This paper is a modified version of the second chapter of the $\mathrm{PhD}$ dissertation of the first author at University of Chile. We thank the co-editor, Satoru Takahashi, two anonymous referees, Nicolás Figueroa, Daniel Hojman, Rahmi Ilkilic, Antonio Romero-Medina, and Juan Pablo Torres-Martínez for useful comments. In particular, we thank a referee for suggesting the preference profile employed in Example 3 and spotting mistakes of previous versions of the paper. Fonseca-Mairena and Triossi acknowledge financial support from the Institute for Research in Market Imperfections and Public Policy, ICM IS130002, Ministerio de Economía, Fomento y Turismo. Triossi acknowledges financial support from Ca' Foscari University of Venice under project MAN.INS_TRIOSSI and from Ministerio de Ciencia e Innovación under project AEI PID2020-118022GB-I00. The authors declare they do not have any conflict of interest.

${ }^{\dagger}$ Corresponding author. Department of Economics and Management, Universidad Católica del Maule; Observatorio Laboral del Maule. San Miguel 3605, Talca, Chile. E-mail: mfonseca@ucm.cl.

${ }^{\ddagger}$ Department of Management, Ca’ Foscari University of Venice. Fondamenta San Giobbe, Cannaregio 873, 30121 Venice, Italy. E-mail: matteo.triossi@unive.it. 


\section{Introduction}

We study the non-cooperative implementation of cooperative solutions to allocation problems with externalities. That is, we are interested in environments in which the agents care not only about what they receive, but also about what other agents get. An example is the mask allocation in the midst of a viral pandemic where the use of the masks reduces contagion (see, among others Prather et al., 2020). Other relevant examples include housing markets and roommate problems in which the agents are concerned also about their potential neighbors, labor markets in which workers care about their colleagues, and school choice problems in which families care about their children's classmates.

We are interested in implementing social choice rules that are efficient and guarantee individual participation. Among those, the core is of focal importance in cooperative game theory. An allocation is in the core if it is resistant to group deviations. Sönmez (1999) proves that a strategy-proof and individually rational social choice function exists if and only if the core is essentially single valued, whenever the core is nonempty. Unfortunately, the core is often empty in markets with externalities. The reason is that the standard definition of blocking assumes that, when a coalition deviates, the agents outside the coalition will not react. The assumption is overly optimistic from the point of view of the deviating agents and multiplies the number of blocking coalitions. ${ }^{1}$ In our model, the members of a coalition do not make any assumption about the goods assigned to the agents outside the coalition and they act prudently. More precisely, we assume that a coalition blocks an allocation if each member of the coalition prefers the worst allocation consistent with the deviation to the proposed allocation. ${ }^{2}$ We call this kind of blocking $\alpha$-blocking and the $\alpha$-core is the set of $\alpha$-unblocked allocations. ${ }^{3}$ The $\alpha$-core has been introduced in Aumann and Peleg (1960) and Aumann (1961). ${ }^{4}$ Several works have as-

\footnotetext{
${ }^{1}$ Ehlers (2018) extends the analysis by Sönmez (1999) to environments with externalities and prove that, whenever the individually rational core is nonempty, a strategy-proof and individually rational social choice function exists if and only if the individually rational core is essentially single valued.

${ }^{2}$ Prudent behavior is closely related to maxmin behavior. Barberà and Dutta $(1982,1995)$ study protective behavior which refines the traditional maxmin behavior.

${ }^{3}$ The $\alpha$-core equals the core if there are not externalities.

${ }^{4}$ Kajii (1992) proves existence results for $\alpha$-cores in normal form games with externalities, see also Martins-da-Rocha and Yannelis (2011) and Basile and Scalzo (2020).
} 
sumed prudent behavior for contexts with externalities. Among them, Sasaki and Toda (1996) and Contreras and Torres-Martínez (2021) study pairwise stability in marriage markets with externalities and in roommate problems with externalities, respectively. First, we study implementation in dominant strategies. Hong and Park (2018) consider a model with externalities and prove that if the agents care about their allocation prior to the other agents', the Top Trading Cycle (TTC from now on) generates an efficient, individually rational, and strategy-proof, social choice function. We prove that their result do not generalize to arbitrary externalities: no $\alpha$-individually rational and efficient mechanism is implementable in dominant strategies in allocation problems with externalities.

This negative result leads us to consider the implementation of the $\alpha$-core correspondence in Nash equilibrium. This research question relates to Sönmez (1996) who shows that the core correspondence is implementable in Nash equilibrium in a class of assignment problems in which the core is nonempty and agents have strict preferences over the objects, thus without externalities. Kara and Sönmez $(1996,1997)$ prove the Nash implementability of stable (and thus core) solutions in one-to-one and many-to-one matching markets, respectively, under strict preferences over partners. Fonseca-Mairena and Triossi (2019) prove the Nash implementability of stable solutions in one-to-one matching markets with externalities, in which pairs act prudently when deviating. In the last case, stable allocations are not necessarily efficient and thus do not belong to the core.

We prove that the $\alpha$-core is not implementable in Nash equilibrium under general preferences. Restricting our attention to the domain $\widetilde{\mathcal{R}}$ in which the $\alpha$-core equals the weak $\alpha$-core and both are nonempty, we prove that the $\alpha$-core correspondence is implementable in Nash equilibrium. When preferences are strict the $\alpha$-core equals the weak $\alpha$-core. This condition is satisfied, for example, in the marriage market with externalities studied in Sasaki and Toda (1996), the roommate problem with externalities studied in Contreras and Torres-Martínez (2021), and the housing market with externalities studied in Mumcu and Saglam (2007), among others. The equality between the $\alpha$-core and the weak $\alpha$-core is also satisfied in marriage markets and roommate problems without externalities and 
strict preferences over agents (see Sönmez, 1996). Our result thus applies to all such markets when the $\alpha$-core is nonempty. Also, relaxing the efficiency requirement to weak efficiency, we prove the Nash implementability of the weak $\alpha$-core correspondence on the domain of preference in which it is nonempty.

We then study the limits of implementation. In the subdomain of $\widetilde{\mathcal{R}}$ in which the preferences are strict the $\alpha$-individually rational and efficient social choice rule is implementable in Nash equilibrium. We prove that this is no longer true in $\widetilde{\mathcal{R}}$. In this case, the $\alpha$-core is the maximal implementable, $\alpha$-individually rational, and efficient social choice rule with domain $\widetilde{\mathcal{R}}$.

Finally, we prove that the $\alpha$-core is the minimal monotonic (and thus implementable), $\alpha$-individually rational, and efficient solution, under an additional assumption which limits the externalities that can occur when agents keep their endowments. Thus, under this assumption, the $\alpha$-core is the unique implementable, $\alpha$-individually rational, and efficient social choice rule with domain $\widetilde{\mathcal{R}}$. We also consider the limits of implementation of $\alpha$-individually rational and weakly efficient social choice rules.

The paper is organized as follows. In Section 2 we introduce the model. In Section 3 we consider implementation in dominant strategies. In Section 4 we study implementation in Nash equilibrium. In Section 5 we conclude. The Appendix includes complementary results and examples about the environments we consider.

\section{The Model}

An allocation problem is a quadruple $\left(N,\left(e_{i}\right)_{i \in N}, \mathcal{A}^{f},\left(R_{i}\right)_{i \in N}\right) . N$ is the finite set of agents, we assume $n=|N| \geq 3 .^{5}$ For every $i \in N, e_{i}$ is the endowment of agent $i$, which is the finite set of objects that initially belong to $i$. We assume $e_{i} \cap e_{j}=\emptyset$ for all $i, j \in N, i \neq j$. An allocation is a correspondence $a: N \rightrightarrows \bigcup_{i \in N} e_{i}$ such that for all $x \in \bigcup_{i \in N} e_{i},\left|a^{-1}(x)\right|=1$. We identify an allocation $a$ with the $n$-tuple $(a(i))_{i \in N}$. Let $i \in N$. We denote by $a(-i)$, the $(n-1)$-tuple $(a(j))_{j \in N \backslash\{i\}}$. With abuse of notation, we write $a=(a(i), a(-i))$. Let $\mathcal{A}^{f}$ be the set of feasible allocations, which is a subset of all

\footnotetext{
${ }^{5}|N|$ denotes the cardinality of $N$.
} 
possible allocations $\mathcal{A}$.

Remark 1 The model is able to describe several allocation problems such as:

(i) Housing markets. Allocation problem $\left(N,\left(e_{i}\right)_{i \in N}, \mathcal{A}^{f},\left(R_{i}\right)_{i \in N}\right)$ is a housing market if $\left|e_{i}\right|=1$ for all $i \in N$ and $\mathcal{A}^{f}=\{a \in \mathcal{A}|| a(i) \mid=1, \forall i \in N\}$.

(ii) Roommate problems. Allocation problem $\left(N,\left(e_{i}\right)_{i \in N}, \mathcal{A}^{f},\left(R_{i}\right)_{i \in N}\right)$ is a roommate problem if $e_{i}=\{i\}$ for all $i \in N$ and $\mathcal{A}^{f}=\left\{a \in \mathcal{A} \mid a(i)=e_{j} \Leftrightarrow a(j)=e_{i}, \forall i, j \in N\right\}$.

(iii) Marriage markets. Allocation problem $\left(N,\left(e_{i}\right)_{i \in N}, \mathcal{A}^{f},\left(R_{i}\right)_{i \in N}\right)$ is a marriage market if there exist two subsets $W$ and $M$ such that $W \cup M=N$ and $W \cap M=\emptyset$, $e_{i}=\{i\}$ for all $i \in N$, and $\mathcal{A}^{f}=\{a \in \mathcal{A} \mid \forall i \in W, \forall j \in M, a(i) \in M \cup\{i\}, a(j) \in$ $\left.W \cup\{j\}, a(i)=e_{j} \Leftrightarrow a(j)=e_{i}\right\} .6,7$

For every $i \in N, R_{i}$ is the preference relation of agent $i$, which is a reflexive, complete, and transitive relation on $\mathcal{A}^{f}$. Let $e$ be the allocation in which each agent keeps her endowment which is $e(i)=e_{i}$ for all $i \in N$. We assume $e \in \mathcal{A}^{f}$. Let $A_{i}=\left\{a \in \mathcal{A}^{f} \mid a(i)=e_{i}\right\}$ be the set of allocations in which agent $i$ keeps her endowment. Let $R=\left(R_{i}\right)_{i \in N}$. We say that $R_{i}$ is strict if, for all $a, b \in \mathcal{A}^{f}, a R_{i} b$ and $b R_{i} a$ implies $a=b$. We say that $R$ is strict if $R_{i}$ is strict for all $i \in N$. For all $a, b \in \mathcal{A}^{f}$, by $a P_{i} b$ we mean $a R_{i} b$ and $\neg b R_{i} a$, by $a I_{i} b$ we mean $a R_{i} b$ and $b R_{i} a$. We denote by $\mathcal{R}$ the set of all preferences over $\mathcal{A}^{f}$ and by $\mathcal{P} \subseteq \mathcal{R}$ the set of strict preferences over $\mathcal{A}^{f}$. An allocation problem has externalities if there exists $i \in N$ and $a, b \in \mathcal{A}^{f}$ such that $a(i)=b(i)$ and $a P_{i} b$, that is, even though agent $i$ gets the same object in $a$ and in $b$, she strictly prefers $a$. Consider a preference domain, $\mathcal{D} \subseteq \mathcal{R}^{|N|}$. A social choice function ( $S C F$ from now on) $\Gamma: \mathcal{D} \rightarrow \mathcal{A}^{f}$ associates to each preference profile an allocation. A social choice rule ( $S C R$ from now on) is a nonempty correspondence $\Omega: \mathcal{D} \rightrightarrows \mathcal{A}^{f}$, which associates to each preference profile a nonempty subset of allocations. ${ }^{8}$ The restriction of a $S C R, \Omega$ to a nonempty subdomain $\mathcal{D}^{\prime} \subseteq \mathcal{D}$ is

\footnotetext{
${ }^{6}$ Notice that a marriage market is a special kind of a roommate problem, and a roommate problem is a special kind of a housing market.

${ }^{7}$ Alternatively, housing markets, roommate problems, and marriage markets can be defined as restrictions on admissible preference profiles.

${ }^{8}$ Formally, a correspondence $\Omega$ is nonempty if $\Omega(R) \neq \emptyset$ for all $R \in \mathcal{D}$.
} 
the $S C R, \Omega_{\mid \mathcal{D}^{\prime}}: \mathcal{D}^{\prime} \rightrightarrows \mathcal{A}^{f}$ in which $\Omega_{\mid \mathcal{D}^{\prime}}(R)=\Omega(R)$ for all $R \in \mathcal{D}^{\prime}$. The restriction of a $S C F$ to a nonempty subdomain is defined analogously.

\subsection{The core and the $\alpha$-core}

Let $\mathcal{D} \subseteq \mathcal{R}^{|N|}$ be a preference domain and let $\left(N,\left(e_{i}\right)_{i \in N}, \mathcal{A}^{f},\left(R_{i}\right)_{i \in N}\right)$ be an allocation problem in which $R=\left(R_{i}\right)_{i \in N} \in \mathcal{D}$. We consider allocations that are not blocked by coalitions of agents. The standard definition of a blocking coalition is as follows: coalition $T$ blocks allocation $a \in \mathcal{A}^{f}$ if there exists $b \in \mathcal{A}^{f}$ such that: $(i) \bigcup_{i \in T} b(i)=\bigcup_{i \in T} e_{i} ;(i i)$ $b R_{i} a$ for all $i \in T$; $(i i i) b P_{j} a$ for some $j \in T$. An allocation is in the core if it is not blocked by any coalition. If we replace $(i i)$ and $(i i i)$ by $(i i)^{\prime} b P_{i} a$ for all $i \in T$, we say that coalition $T$ strongly blocks allocation $a$. An allocation is in the weak core if it is not strongly blocked by any coalition. For each $R \in \mathcal{D}$, we denote by $\mathscr{C}(R)$ and $\mathscr{W} \mathscr{C}(R)$, the core and the weak core, respectively. Let $\mathscr{C}: \mathcal{D} \rightrightarrows \mathcal{A}^{f}$ and $\mathscr{W} \mathscr{C}: \mathcal{D} \rightrightarrows \mathcal{A}^{f}$ be the core correspondence and the weak core correspondence, respectively. Allocation $a$ is individually rational if it is not individually blocked, which is if $a R_{i} b$ for all $b \in A_{i}$, for all $i \in N .^{9}$ For each $R \in \mathcal{D}$, we denote the set of feasible allocations that are individually rational by $\mathscr{I}(R)$.

The set of individually rational allocations and thus the core can be empty if there are externalities.

Example 1 Let $N=\{1,2,3\}$. Let $\mathcal{A}^{f}=\{a, b, e\}$, where $a=\left(e_{1}, e_{3}, e_{2}\right), b=\left(e_{3}, e_{2}, e_{1}\right)$, and $e=\left(e_{1}, e_{2}, e_{3}\right) \cdot{ }^{10}$ Let $a P_{1} b P_{1} e, b P_{2} a P_{2} e$, and $a P_{3} b P_{3} e$. There is no individually rational allocation because agent 1 blocks all allocations except a and agent 2 blocks a.

When there are externalities, the number of blocking coalitions is potentially very large then the core is often empty. In general, blocking decisions should depend on the ex-

\footnotetext{
${ }^{9}$ In this paper, individual rationality is a participation constraint on allocations. If the market does not have externalities, it can be interpreted simultaneously as a participation constraint and a normative welfare requirement: the proposed allocation should not decrease any agent's initial utility. In market with externalities this latter requirement reads as $a R_{i} e$ for all $i \in N$, which is not equivalent to the participation constraint. It is the definition of individual rationality employed by Sönmez (1999) and Ehlers (2018). However, in both papers, the restrictions on admissible preference domains (Condition 1 in both papers) make the participation constraint and the welfare condition equivalent.

${ }^{10}$ For example, by $a=\left(e_{1}, e_{3}, e_{2}\right)$ we mean that agent 1 keeps her endowment while agents 2 and 3 exchange their respective endowments.
} 
pectations about the reaction of the other agents to coalitional deviations. We assume that the agents are not able to make any prediction about the behavior of other agents. Thus, a coalition $T$ blocks an allocation if the payoff received by every member of $T$ from deviating is superior to the payoff from implementing the allocation, independently on the bundle of goods the agents outside the coalition get, and for at least one member that payoff is strictly superior. Formally, coalition $T \subseteq N \boldsymbol{\alpha}$-blocks allocation $a \in \mathcal{A}^{f}$ if there exists $b \in \mathcal{A}^{f}$ such that: $(i) \bigcup_{i \in T} b(i)=\bigcup_{i \in T} e_{i} ;(i i) c R_{i} a$ for each $i \in T$ and for all $c \in \mathcal{A}^{f}$ such that $c(i)=b(i) \forall i \in T ;($ iii $)$ there exists $j \in T$ such that $c P_{j} a$ for all $c \in \mathcal{A}^{f}$ such that $c(i)=b(i) \forall i \in T$. Allocation $a$ belongs to the $\boldsymbol{\alpha}$-core if it is not $\alpha$-blocked by any coalition. If we replace conditions $(i i)$ and $(i i i)$ by $(i i)^{\prime} c P_{i} a$ for each $i \in T$ and for all $c \in \mathcal{A}^{f}$ such that $c(i)=b(i) \forall i \in T$, we say that coalition $T$ strongly $\boldsymbol{\alpha}$-blocks allocation $a$. An allocation is in the weak $\boldsymbol{\alpha}$-core if it is not strongly $\alpha$-blocked by any coalition. For each $R \in \mathcal{D}$, we denote by $\alpha \mathscr{C}(R)$ and $\mathscr{W} \alpha \mathscr{C}(R)$, the $\alpha$-core and the weak $\alpha$-core, respectively. Let $\alpha \mathscr{C}: \mathcal{D} \rightrightarrows \mathcal{A}^{f}$ and $\mathscr{W} \alpha \mathscr{C}: \mathcal{D} \rightrightarrows \mathcal{A}^{f}$ be the $\alpha$-core correspondence and the weak $\alpha$-core correspondence, respectively. Allocation $a$ is $\boldsymbol{\alpha}$-individually rational if it is not $\alpha$-blocked by any agent, which is if, for each $i \in N, a R_{i} b$ for some $b \in A_{i}$. For each $R \in \mathcal{D}$, we denote the set of feasible allocations that are $\alpha$-individually rational by $\alpha \mathscr{I}(R)$. The set $\alpha \mathscr{I}(R) \neq \emptyset$ because $e \in \mathcal{A}^{f}$ by assumption. A $S C R \Omega: \mathcal{D} \rightrightarrows \mathcal{A}^{f}$ is $\alpha$-individually rational if $a \in \alpha \mathscr{I}(R)$ for all $a \in \Omega(R)$ and for all $R \in \mathcal{D}$.

The next result establishes relations between the different core notions.

Proposition 1 Let $R \in \mathcal{R}^{|N|}$, then

(a) $\mathscr{C}(R) \subseteq \mathscr{W} \mathscr{C}(R)$. If $R$ is strict $\mathscr{C}(R)=\mathscr{W} \mathscr{C}(R)$

(b) $\alpha \mathscr{C}(R) \subseteq \mathscr{W} \alpha \mathscr{C}(R)$. If $R$ is strict $\alpha \mathscr{C}(R)=\mathscr{W} \alpha \mathscr{C}(R)$.

(c) $\mathscr{C}(R) \subseteq \alpha \mathscr{C}(R)$. If there are not externalities $\mathscr{C}(R)=\alpha \mathscr{C}(R)$.

(d) $\mathscr{W} \mathscr{C}(R) \subseteq \mathscr{W} \alpha \mathscr{C}(R)$. If there are not externalities $\mathscr{W} \mathscr{C}(R)=\mathscr{W} \alpha \mathscr{C}(R)$

The proof of the result is straightforward and thus omitted. 
A feasible allocation $a$ is efficient under $R$ if there is no $b \in \mathcal{A}^{f}$ such that $b R_{i} a$ for all $i \in N$ and $b P_{j} a$ for some $j \in N$. Let $\mathscr{E}(R)$ be the set of efficient allocations under $R$. The set of efficient allocations is the set of allocation that are not blocked by the grand coalition, $N$. A feasible allocation $a$ is weakly efficient under $R$ if there is no $b \in \mathcal{A}^{f}$ such that $b P_{i} a$ for all $i \in N$. Let $\mathscr{W} \mathscr{E}(R)$ be the set of weakly efficient allocations under $R$. Since blocking and $\alpha$-blocking by the grand coalition coincide, both the core and the $\alpha$-core are subsets of the set of efficient allocations. Formally, $\mathscr{C}(R) \cup \alpha \mathscr{C}(R) \subseteq \mathscr{E}(R)$ for all $R \in \mathcal{R}^{|N|}$. However, the weak core and the weak $\alpha$-core may not satisfy efficiency. Still, the weak core and the weak $\alpha$-core are subsets of the set weakly efficient allocations. Formally, $\mathscr{W} \mathscr{C}(R) \cup \mathscr{W} \alpha \mathscr{C}(R) \subseteq \mathscr{W} \mathscr{E}(R)$ for all $R \in \mathcal{R}^{|N|}$. A $S C R \Omega: \mathcal{D} \rightrightarrows \mathcal{A}^{f}$ is efficient if $a \in \mathscr{E}(R)$ for all $a \in \Omega(R)$ and for all $R \in \mathcal{D} ; \Omega$ is weakly efficient if $a \in \mathscr{W} \mathscr{E}(R)$ for all $a \in \Omega(R)$ and for all $R \in \mathcal{D}$. Let $\alpha \mathscr{I} \mathscr{E}(R)=\alpha \mathscr{I}(R) \cap \mathscr{E}(R)$ be the set of $\alpha$-individually rational and efficient allocations under $R$. Let $\alpha \mathscr{I} \mathscr{E}$ be the $\alpha$-individually rational and efficient correspondence $\alpha \mathscr{I} \mathscr{E}: \mathcal{D} \rightrightarrows \mathcal{A}^{f}$. Let $\alpha \mathscr{I} \mathscr{W} \mathscr{E}(R)=\alpha \mathscr{I}(R) \cap \mathscr{W} \mathscr{E}(R)$ be the set of $\alpha$-individually rational and weakly efficient allocations under $R$. Let $\alpha \mathscr{I} \mathscr{W} \mathscr{E}$ be the $\alpha$-individually rational and weakly efficient correspondence $\alpha \mathscr{I} \mathscr{W} \mathscr{E}: \mathcal{D} \rightrightarrows \mathcal{A}^{f}$.

\subsection{Implementation}

For each $i \in N$ let $\mathcal{S}_{i} \subseteq \mathcal{R}$. Let $\mathcal{D}=\prod_{i \in N} \mathcal{S}_{i}$. A $S C F \Gamma: \mathcal{D} \rightarrow \mathcal{A}^{f}$ is implementable in dominant strategies (or strategy-proof) if $\Gamma(R) R_{i} \Gamma\left(\widetilde{R}_{i}, R_{-i}\right)$ for all $i \in N$, $R \in \mathcal{D}$, and $\widetilde{R}_{i} \in \mathcal{S}_{i}$.

Now, for each $i \in N$ let $S_{i}$ be the strategy space of agent $i$. A mechanism is a pair $(S, g)$ where $S=\prod_{i \in N} S_{i}$ is the strategy space and $g: S \rightarrow \mathcal{A}^{f}$ is the outcome function. A mechanism $(S, g)$ induces a strategic form game, $(N, R, S, g)$. Let $N E(R, S, g)$ be the set of pure strategy Nash equilibria of game $(N, R, S, g)$. Let $\Omega$ be a $S C R$. Mechanism $(S, g)$ implements $\Omega$ in Nash equilibrium if $g(N E(R, S, g))=\Omega(R)$ for all $R \in \mathcal{D}$ (see Maskin, 1999).

Remark 2 The definition of implementation in Nash equilibrium implies that, if a SCR $\Omega: \mathcal{D} \rightrightarrows \mathcal{A}^{f}$ is implementable in Nash equilibrium, the restriction of $\Omega$ to any nonempty 
subdomain $\mathcal{D}^{\prime} \subseteq \mathcal{D}$ is implementable in Nash equilibrium as well.

\section{Implementation in dominant strategies: an impos- sibility result}

In house allocation problems without externalities, the Top Trading Cycles mechanism (Shapley and Scarf, 1974; Roth and Postlewaite, 1977) is an individually rational and efficient mechanism. The TTC works as follows: 1. Each agent $i$ points at her "top (most preferred)" house. Draw an arrow from each agent $i$ to the agent who holds the top house of $i$. 2. There is at least one cycle in the graph. Reallocate each house to the agent belonging to the cycle pointing to it and remove all the involved agents and houses from the graph. 3. If there are remaining agents, go back to step 1, otherwise stop.

Hong and Park (2018) consider housing markets (see Remark 1) and assume agents primarily care about their own allocation. In this case, it is possible to infer preferences over the objects from the preferences over the allocations in a univocal way. Formally, they assume that, for all $a, b \in \mathcal{A}^{f}:(i) a I_{i} b \Rightarrow a(i)=b(i) ;(i i)$ if $a(i) \neq b(i)$, then $a P_{i} b \Leftrightarrow$ $(a(i), c(-i)) P_{i}(b(i), d(-i))$ for all $c(-i), d(-i)$ such that $(a(i), c(-i)),(b(i), d(-i)) \in$ $\mathcal{A}^{f}$. They call this kind of preferences "egocentric". Egocentric preferences naturally in-

duce the following preference profile over the objects: if $a(i) \neq b(i), a(i) \widehat{P}_{i} b(i) \Leftrightarrow$ $(a(i), a(-i)) P_{i}(b(i), b(-i))$ for some $a(-i), b(-i)$ such that $(a(i), a(-i)),(b(i), b(-i)) \in$ $\mathcal{A}^{f}$. Hong and Park (2018) prove that performing the TTC with the induced preferences generates a mechanism which is efficient, individually rational (thus $\alpha$-individually rational), and strategy proof. However, there is no straightforward way to extend TTC to environments with arbitrary externalities, even for slight departures from the egocentric model as shown in Example 2.

Example 2 Let $N=\{1,2,3\}$. Let $\mathcal{A}^{f}=\{a, b, c, d, e, f\}$, where $a=\left(e_{1}, e_{3}, e_{2}\right), b=$ $\left(e_{2}, e_{1}, e_{3}\right), c=\left(e_{2}, e_{3}, e_{1}\right), d=\left(e_{3}, e_{1}, e_{2}\right), e=\left(e_{1}, e_{2}, e_{3}\right)$, and $f=\left(e_{3}, e_{2}, e_{1}\right)$. Consider 
the following preferences:

$$
R_{1}: c P_{1} b P_{1} d P_{1} f P_{1} a P_{1} e ; \quad R_{2}: c P_{2} a P_{2} b P_{2} d P_{2} f P_{2} e ; \quad R_{3}: d P_{3} a P_{3} c P_{3} f P_{3} b P_{3} e .
$$

The preference profile $\left(R_{i}\right)_{i \in N}$ is egocentric and induces the following preferences over the objects:

$$
\widehat{R}_{1}: e_{2} \widehat{P}_{1} e_{3} \widehat{P}_{1} e_{1} ; \quad \widehat{R}_{2}: e_{3} \widehat{P}_{2} e_{1} \widehat{P}_{2} e_{2} ; \quad \widehat{R}_{3}: e_{2} \widehat{P}_{3} e_{1} \widehat{P}_{3} e_{3}
$$

Applying the TTC to $\left(\widehat{R}_{i}\right)_{i=1}^{3}$ yields agents 1 and 3 pointing at $e_{2}$, agent 2 pointing at $e_{3}$. There is only one cycle between agents 2 and 3 . The final allocation is $\left(e_{1}, e_{3}, e_{2}\right)=a$. The reader can easily check that no agents has incentives in misrepresenting the induced preferences over the objects and that allocation a is efficient and individually rational. We now modify the preferences of agent 3 into $\widetilde{R}_{3}$ : d $\widetilde{P}_{3} c \widetilde{P}_{3} a \widetilde{P}_{3} f \widetilde{P}_{3} b \widetilde{P}_{3}$ e. Let $\widetilde{R}=$ $\left(R_{1}, R_{2}, \widetilde{R}_{3}\right)$ There is no unequivocal way to transform the preferences of agent 3 into preferences over objects. For example, assume agent 3 compares among the worst alternatives. The worst allocations where she gets $e_{1}, e_{2}$, and $e_{3}$ are $f$, a, and e, respectively. Since a $\widetilde{P}_{3} f \widetilde{P}_{3}$ e, then the induced preferences over objects would be $\widehat{R}_{3}: e_{2} \widehat{P}_{3} e_{1} \widehat{P}_{3} e_{3} \cdot{ }^{11} A p$ plying TTC with preferences $\left(\widehat{R}_{i}\right)_{i=1}^{3}$ yields a, which is not efficient under $\widetilde{R}$ since all agents strictly prefer $c$ to $a$.

In general, no strategy-proof, $\alpha$-individually rational, and efficient mechanism exists if the set of admissible preferences of each agent includes all strict preferences.

Proposition 2 Assume $\left|\mathcal{A}^{f}\right| \geq 3$. Let $\mathcal{P} \subseteq \mathcal{S}_{i}$ for all $i \in N$ and let $\mathcal{D}=\prod_{i \in N} \mathcal{S}_{i}$. There is no strategy-proof, $\alpha$-individually rational, and efficient $S C F, \Gamma: \mathcal{D} \rightarrow \mathcal{A}^{f}$.

Proof. We prove the claim by contradiction. Assume mechanism $\Gamma: \mathcal{D} \rightarrow \mathcal{A}^{f}$ is a strategy-proof, $\alpha$-individually rational, and efficient mechanism. Let $\Gamma^{\prime}=\Gamma_{|\mathcal{P}| N \mid}$. Then $\Gamma^{\prime}$ is the restriction of $\Gamma$ to the domain of strict preference profiles. Thus, $\Gamma^{\prime}$ is strategy-proof, $\alpha$-individually rational, and efficient. Since $\Gamma^{\prime}$ is efficient, then $\Gamma^{\prime}\left(\mathcal{P}^{|N|}\right)=\mathcal{A}^{f}$. Then, by strategy proofness, the Gibbard-Satterthwaite theorem (Gibbard, 1973; Satterthwaite,

\footnotetext{
${ }^{11}$ Notice that if agent 3 compared among the best alternatives we would obtain $\widehat{R}_{3}$ as well.
} 
1975) implies that there exists a dictator, $j \in N$. Let $a \in \mathcal{A}^{f} \backslash\{e\}$ and let $\widetilde{R} \in \mathcal{P}^{|N|}$ such that:

(i) $a \widetilde{P}_{j} b$ for all $b \neq a$;

(ii) $b \widetilde{P}_{k} a$ for all $b \neq a$, for all $k \neq j$.

Then $\Gamma^{\prime}(\widetilde{R})=a$ because $j$ is the dictator. Since $a \neq e$, there exists $k \neq j$ such that $a(k) \neq e_{k}$. Then, by $(i i)$, agent $k \alpha$-blocks $a$, which leads to a contradiction.

The Gibbard-Satterthwaite theorem implies that any strategy-proof and efficient mechanism is dictatorial. In order to complete the proof of Proposition 2, we show that, under externalities, any dictatorial rule is not $\alpha$-individually rational.

The proof of the result implies that in any efficient and strategy-proof mechanism, some agent will be strictly better off by keeping her endowment rather than participating to the redistribution, independently on the behavior of the other agents. Also, the results does not change if we replace $\alpha$-individual rationality by individual rationality since the latter condition is stronger.

If there are not externalities the impossibility result does not hold. As already observed, in house assignment problems, the TTC is an individually rational, efficient, and strategyproof mechanism.

\section{Implementation in Nash equilibrium}

In this section we relax the equilibrium requirement and study the possibility of implementing efficient and $\alpha$-individually rational correspondences in $N E$. We start introducing additional notation and results. Let $i \in N$ and let $R_{i}$ preferences over $\mathcal{A}^{f}$. Let $L\left(a, R_{i}\right)=\left\{b \in \mathcal{A}^{f}: a R_{i} b\right\}$ be the lower contour set of $a \in \mathcal{A}^{f}$ at $R_{i}$. A $S C R$, $\Omega: \mathcal{D} \rightrightarrows \mathcal{A}^{f}$ is (Maskin) monotonic if, for all $R, R^{\prime} \in \mathcal{D}$ and all $a \in \mathcal{A}^{f}$ such that $a \in \Omega(R)$ and $L\left(a, R_{i}\right) \subseteq L\left(a, R_{i}^{\prime}\right)$ for all $i \in N$, then $a \in \Omega\left(R^{\prime}\right)$. Let $i \in N$ and $X \subseteq \mathcal{A}^{f}$. Monotonicity is a necessary condition for implementation in $N E$, but it is not, in general, a sufficient one (see Maskin, 1999). 
An allocation $a \in X$ is essential for agent $i \in N$ in the set $X$ for $\Omega$ if $a \in \Omega(R)$ for some preference profile $R$ such that $L\left(a, R_{i}\right) \subseteq X$. The set of essential allocations is denoted by $\operatorname{Ess}(\Omega, i, X)$. Correspondence $\Omega$ is essentially monotonic if for all $R, R^{\prime} \in \mathcal{D}$, for all $a \in \Omega(R)$, if $\operatorname{Ess}\left(\Omega, i, L\left(a, R_{i}\right)\right) \subseteq L\left(a, R_{i}^{\prime}\right)$ for all $i \in N$, then $a \in \Omega\left(R^{\prime}\right)$. Assume $\Omega$ is essentially monotonic and assume allocation $a$ is chosen by $\Omega$ when the preference profile is $R$. If passing from profile $R$ to profile $R^{\prime}, a$ does not worsen its ranking with respect to essential allocations, alternative $a$ is chosen when the preference profile is $R^{\prime}$, too. ${ }^{12}$ Thus, essential monotonicity is stronger than monotonicity.

When there are at least three agents, an essentially monotonic correspondence is implementable in Nash equilibrium (Yamato 1992, Theorem 2, p. 488, see also Remark 2, p. 490).

In environments without externalities, Sönmez (1996) shows that the core correspondence is the minimal individually rational, efficient, and monotonic solution if the preferences over the objects are strict. Furthermore he proves that it is implementable in $N E$ on the domain in which it is nonempty. In Proposition 3 we show that the result does not extend to market with externalities. More precisely, we prove that the core and the $\alpha$-core are not implementable in $N E$ on the domains in which they are nonempty. Let $\mathcal{R}_{1}=\left\{R \in \mathcal{R}^{|N|} \mid \mathscr{C}(R) \neq \emptyset\right\}$ and $\mathcal{R}_{2}=\left\{R \in \mathcal{R}^{|N|} \mid \alpha \mathscr{C}(R) \neq \emptyset\right\}$.

Proposition 3 Let $\left|\mathcal{A}^{f}\right| \geq 3$. Social choice rules $\mathscr{C}_{\mid \mathcal{R}_{1}}$ and $\alpha \mathscr{C}_{\mid \mathcal{R}_{2}}$ are not implementable in $N E$.

Proof. By assumption there exist distinct $a, b \in \mathcal{A}^{f} \backslash\{e\}$. Thus, $\{a, b, e\} \subseteq \mathcal{A}^{f}$. Let $k \in N$ such that $b \notin A_{k}$. We know $k$ exists because $b \neq e$. Consider the following preferences: $R_{i}: a P_{i} b P_{i} e P_{i} c$ for all $c \in \mathcal{A}^{f} \backslash\{a, b, e\}$ and for all $i \in N \backslash\{k\} ; b P_{k} a P_{k} e P_{k} c$ for all $c \in \mathcal{A}^{f} \backslash\{a, b, e\}$. Let $R=\left(R_{i}\right)_{i \in N}$. Since $a \in \mathscr{C}(R) \subseteq \alpha \mathscr{C}(R), R \in \mathcal{R}_{1} \cap \mathcal{R}_{2}$. Let $R_{i}^{\prime}: a I_{i}^{\prime} b P_{i}^{\prime} e P_{i}^{\prime} c$ for all $c \in \mathcal{A}^{f} \backslash\{a, b, e\}$ and for all $i \in N \backslash\{k\}$. Let $R_{k}^{\prime}=R_{k}$ and let $R^{\prime}=\left(R_{i}^{\prime}\right)_{i \in N}$. We have $L\left(a, R_{i}\right)=L\left(a, R_{i}^{\prime}\right)$ for all $i \in N$. Since $b \in \mathscr{C}\left(R^{\prime}\right) \subseteq \alpha \mathscr{C}\left(R^{\prime}\right)$, $R^{\prime} \in \mathcal{R}_{1} \cap \mathcal{R}_{2}$. However, $a \notin \mathscr{C}\left(R^{\prime}\right)=\alpha \mathscr{C}\left(R^{\prime}\right)$ because the grand coalition $N$ blocks

\footnotetext{
${ }^{12}$ In Yamato (1992), essential monotonicity is called "strong monotonicity".
} 
$a$ by announcing $b$. It follows that $\mathscr{C}_{\mid \mathcal{R}_{1}}$ and $\alpha \mathscr{C}_{\mid \mathcal{R}_{2}}$ are not monotonic and thus not implementable in $N E$.

The proof of Proposition 3 relies on the existence of profile $R^{\prime}$ for which the $\alpha$-core and the weak $\alpha$-core differ, indeed $a \in \mathscr{W} \alpha \mathscr{C}\left(R^{\prime}\right) \backslash \alpha \mathscr{C}\left(R^{\prime}\right)$.

We thus restrict our attention to preference profiles such that the weak $\alpha$-core and the $\alpha$ core coincide and they are both nonempty which is $\widetilde{\mathcal{R}}=\left\{R \in \mathcal{R}^{|N|} \mid \alpha \mathscr{C}(R)=\mathscr{W} \alpha \mathscr{C}(R) \neq \emptyset\right\}$.

To provide intuition about the structure of $\widetilde{\mathcal{R}}$ we consider marriage markets, housing markets, and roommate problems (Remark 1). ${ }^{13}$ Let $\mathcal{R}^{\text {wes }}$ be the domain of profiles with strict preferences over objects and without externalities, let $\mathcal{R}^{\text {seg }}$ be the domain of profiles of strict egocentric preferences (formal definitions are provided in the Appendix). For roommate problems, let $\mathcal{R}^{\text {wesno }}$ be the set of profiles with strict preferences over objects and without externalities that satisfy the no odd rings condition (Chung, 2000), let $\mathcal{R}^{\text {segno }}$ be the domain of strict egocentric profiles such that the associated profiles without externalities satisfy the no odd rings condition.

Proposition 4 The following claims hold:

(a) In marriage markets $\mathcal{R}^{\text {wes }} \cup \mathcal{R}^{\text {seg }} \subseteq \widetilde{\mathcal{R}}$.

(b) In housing markets $\mathcal{R}^{\text {seg }} \subseteq \widetilde{\mathcal{R}}$ and $\mathcal{R}^{\text {wes }} \cap \widetilde{\mathcal{R}} \neq \emptyset$.

(c) In roommate problems $\mathcal{R}^{\text {wesno }} \cup \mathcal{R}^{\text {segno }} \subseteq \widetilde{\mathcal{R}}$.

The proof of Proposition 4 is in the Appendix.

Proposition 4 does not provide a complete characterization of $\widetilde{\mathcal{R}}$ : all inclusions are strict. Consider Example 1 which describes simultaneously a housing market, a marriage market, and a roommate problem. Here, the allocation problem has externalities and preferences are not egocentric, the core is empty, while the $\alpha$-core and the weak $\alpha$-core coincide, and they are nonempty, so $R \in \widetilde{\mathcal{R}} \backslash\left(\mathcal{R}^{\text {wes }} \cup \mathcal{R}^{\text {seg }}\right)$.

Furthermore there are other profiles belonging to $\widetilde{\mathcal{R}}$. For example, in marriage markets with externalities, if the (strict) preferences weakly agree over a top-ranked collection of

\footnotetext{
${ }^{13}$ Recall that an allocation problem is determined by the set of agents $N$, the set of feasible allocations $\mathcal{A}^{f}$, the endowments of the agents $\left(e_{i}\right)_{i \in N}$, and the preferences of the agents $R=\left(R_{i}\right)_{i \in N}$. It follows that $\widetilde{\mathcal{R}}$ depends on the set of agents, their endowments, and the set of feasible allocations.
} 
allocations but sufficiently disagree about the rankings of the members of this collection (Theorem 1 in Mumcu and Saglam, 2010) the $\alpha$-core is nonempty. ${ }^{14}$

\subsection{Implementation of the $\alpha$-core correspondence}

We now present the main result of this section.

Theorem 1 The $\alpha$-core correspondence, $\alpha \mathscr{C}: \widetilde{\mathcal{R}} \rightrightarrows \mathcal{A}^{f}$ is implementable in Nash equilibrium.

We next prove that $\alpha \mathscr{C}$ is implementable in $N E$, by proving that it is essentially monotonic. The result follows from two preliminary results. First, Lemma 1 implies monotonicity is equivalent to essential monotonicity in our environment.

Lemma 1 Let $R \in \widetilde{\mathcal{R}}$. Then Ess $\left(\alpha \mathscr{C}, i, L\left(a, R_{i}\right)\right)=L\left(a, R_{i}\right)$, for all $a \in \alpha \mathscr{C}(R)$.

Proof. Let $i \in N, R \in \widetilde{\mathcal{R}}, a \in \alpha \mathscr{C}(R)$. The proof is in two steps.

(i) We prove $\operatorname{Ess}\left(\alpha \mathscr{C}, i, L\left(a, R_{i}\right)\right) \subseteq L\left(a, R_{i}\right)$. Assume $b \in \operatorname{Ess}\left(\alpha \mathscr{C}, i, L\left(a, R_{i}\right)\right)$. By definition of $E s s\left(\alpha \mathscr{C}, i, L\left(a, R_{i}\right)\right)$, there exists a preference profile $R^{\prime} \in \widetilde{\mathcal{R}}$ such that $b \in \alpha \mathscr{C}\left(R^{\prime}\right)$ and $L\left(b, R_{i}^{\prime}\right) \subseteq L\left(a, R_{i}\right)$. In particular, $b \in L\left(a, R_{i}\right)$.

(ii) We prove $L\left(a, R_{i}\right) \subseteq E s s\left(\alpha \mathscr{C}, i, L\left(a, R_{i}\right)\right)$. Let $b \in L\left(a, R_{i}\right)$. Let $\widehat{R}$ be an arbitrary strict preference relation over $\mathcal{A}^{f}$. Consider the following preference profile $R^{\prime}$ :

$(\alpha)$ for all $j \in N \backslash\{i\}, c, d \in \mathcal{A}^{f} \backslash\{b\}, b P_{j}^{\prime} c$ and $\left[c P_{j}^{\prime} d \Leftrightarrow c P_{j} d\right.$ or $\left[c I_{j} d\right.$ and $\left.\left.c \widehat{P} d\right]\right]$;

$(\beta)$ for all $c, d \in \mathcal{A}^{f} \backslash\{b\}, b P_{i}^{\prime} c \Leftrightarrow c \in L\left(a, R_{i}\right) \backslash\{b\}, c P_{i}^{\prime} b \Leftrightarrow c \notin L\left(a, R_{i}\right)$, and $c P_{i}^{\prime} d \Leftrightarrow c P_{i} d$ or $\left[c I_{i} d\right.$ and $\left.c \widehat{P} d\right]$

$(\gamma)$ for all $j \in N$, for all $c \in \mathcal{A}^{f} c I_{j}^{\prime} c$.

First, we prove that $R^{\prime} \in \widetilde{\mathcal{R}}$. Since $R_{j}^{\prime}$ is strict for all $j \in N, \alpha \mathscr{C}\left(R^{\prime}\right)=\mathscr{W} \alpha \mathscr{C}\left(R^{\prime}\right)$. Next we prove $\alpha \mathscr{C}\left(R^{\prime}\right) \neq \emptyset$. It suffices to show that $b \in \alpha \mathscr{C}\left(R^{\prime}\right)$. We prove the claim by contradiction. Assume coalition $T \subseteq N \alpha$-blocks $b$. Since $R^{\prime}$ satisfies

\footnotetext{
${ }^{14}$ The core defined in the present paper is a strict subset of the core defined in Mumcu and Saglam (2010, p. 155), which is, in turn, a strict subset of the $\alpha$-core.
} 
$(\alpha)$, coalition $T$ does not contain any agent $j \neq i$. From $(\beta)$, if agent $i \alpha$-blocks $b, i \alpha$-blocks $a$ as well, when the preferences are $R$, which contradicts $a \in \alpha \mathscr{C}(R)$. Then $b \in \alpha \mathscr{C}\left(R^{\prime}\right) \neq \emptyset, R^{\prime} \in \widetilde{\mathcal{R}}$, and $L\left(b, R_{i}^{\prime}\right) \subseteq L\left(a, R_{i}\right)$. This implies $b \in$ $\operatorname{Ess}\left(\alpha \mathscr{C}, i, L\left(a, R_{i}\right)\right)$.

Next, in Lemma 2 we prove that the $\alpha$-core correspondence is monotonic.

Lemma 2 The $\alpha$-core correspondence, $\alpha \mathscr{C}: \widetilde{\mathcal{R}} \rightrightarrows \mathcal{A}^{f}$ is monotonic.

Proof. Let $R \in \widetilde{\mathcal{R}}$ and assume $a \in \alpha \mathscr{C}(R)$. Let $R^{\prime} \in \widetilde{\mathcal{R}}$ such that $L\left(a, R_{i}\right) \subseteq L\left(a, R_{i}^{\prime}\right)$ for all $i \in N$. We prove by contradiction that $a \in \alpha \mathscr{C}\left(R^{\prime}\right)$. Assume $a \notin \alpha \mathscr{C}\left(R^{\prime}\right)$. Then, there exist $b \in \mathcal{A}^{f}$ and $T \subseteq N$ such that:

(i) $\bigcup_{i \in T} b(i)=\bigcup_{i \in T} e_{i}$;

(ii) $c P_{i}^{\prime} a$ for each $i \in T$ and for all $c \in \mathcal{A}^{f}$ such that $c(i)=b(i) \forall i \in T$.

Since $L\left(a, R_{i}\right) \subseteq L\left(a, R_{i}^{\prime}\right)$ for all $i \in N, c P_{i}^{\prime} a$ implies $c P_{i} a$. Then, $a \notin \mathscr{W} \alpha \mathscr{C}(R)=$ $\alpha \mathscr{C}(R)$ which yields a contradiction.

Thus, Theorem 1 follows from Lemma 1, Lemma 2, and Theorem 2 in Yamato (1992). Theorem 1 implies that the restriction of $\alpha \mathscr{C}$ to any nonempty subset of $\widetilde{\mathcal{R}}$ is implementable in Nash equilibrium (see Remark 2).

Additionally, the weak $\alpha$-core correspondence is implementable on the domain in which it is nonempty and the $\alpha$-core correspondence is implementable on the domain of strict preferences in which it is nonempty. Let $\widetilde{\mathcal{R}}^{\prime}=\left\{R \in \mathcal{R}^{|N|} \mid \mathscr{W} \alpha \mathscr{C}(R) \neq \emptyset\right\}$ and let $\widetilde{\mathcal{P}}=$ $\left\{R \in \mathcal{P}^{|N|} \mid \alpha \mathscr{C}(R) \neq \emptyset\right\}$.

Proposition 5 The following claims hold:

(a) Social choice rule $\mathscr{W} \alpha \mathscr{C}_{\mid \widetilde{\mathcal{R}}^{\prime}}$ is implementable in Nash equilibrium.

(b) Social choice rule $\alpha \mathscr{C}_{\mid \widetilde{\mathcal{P}}}=\mathscr{W} \alpha \mathscr{C}_{\mid \widetilde{\mathcal{P}}}$ is implementable in Nash equilibrium.

(c) Assume $\left(\mathcal{R}^{\text {wes }} \cup \mathcal{R}^{\text {seg }}\right) \cap \widetilde{\mathcal{R}} \neq \emptyset$. Social choice rule $\alpha \mathscr{C}_{\mid\left(\mathcal{R}^{\text {wes }} \cup \mathcal{R} \text { seg }\right) \cap \widetilde{\mathcal{R}}}=\mathscr{W} \alpha \mathscr{C}_{\mid\left(\mathcal{R}^{\text {wes } \cup \mathcal{R}}{ }^{\text {seg }) \cap \widetilde{\mathcal{R}}}\right.}$ is implementable in Nash equilibrium. 
The proof of $(a)$ is identical to the proof of Theorem 1 and thus omitted. Parts $(b)$ and $(c)$ follow from Theorem 1 and Remark 2. Equalities $\alpha \mathscr{C}_{\mid \widetilde{\mathcal{P}}}=\mathscr{W} \alpha \mathscr{C}_{\mid \widetilde{\mathcal{P}}}$ and $\alpha \mathscr{C}_{\mid\left(\mathcal{R}^{\text {wes }} \cup \mathcal{R}^{\text {seg }}\right) \cap \tilde{\mathcal{R}}}=$ $\mathscr{W} \alpha \mathscr{C}_{\mid\left(\mathcal{R}^{\text {wes }} \cup \mathcal{R}^{\text {seg }}\right) \cap \widetilde{\mathcal{R}}}$ follow from $\widetilde{\mathcal{P}} \subseteq \widetilde{\mathcal{R}}$ and from $\left(\mathcal{R}^{\text {wes }} \cup \mathcal{R}^{\text {seg }}\right) \cap \widetilde{\mathcal{R}} \subseteq \widetilde{\mathcal{R}}$, respectively Proposition 5, (a) implies that the restriction of $\mathscr{W} \alpha \mathscr{C}$ to any nonempty subset of $\widetilde{\mathcal{R}}^{\prime}$ is implementable in Nash equilibrium (see Remark 2).

Applying Propositions 4, 5, and Remark 2 we obtain the following results regarding marriage markets, housing markets, and roommate problems.

Corollary 1 The following claims hold:

(a) In marriage markets, social choice rule $\alpha \mathscr{C}_{\mid \mathcal{R}^{\text {wes }} \cup \mathcal{R}^{\text {seg }}}=\mathscr{W} \alpha \mathscr{C}_{\mid \mathcal{R}^{\text {wes }} \cup \mathcal{R}^{\text {seg }}}$ is implementable in Nash equilibrium.

(b) In housing markets, social choice rule $\alpha \mathscr{C}_{\mid \mathcal{R}^{\text {seg }}}=\mathscr{W} \alpha \mathscr{C}_{\mid \mathcal{R}^{\text {seg }}}$ is implementable in Nash equilibrium.

(c) In roommate problems, social choice rule $\alpha \mathscr{C}_{\mid \mathcal{R}^{\text {wesno }} \cup \mathcal{R}^{\text {segno }}}=\mathscr{W} \alpha \mathscr{C}_{\mid \mathcal{R}^{\text {wesno }} \cup \mathcal{R}^{\text {segno }}}$ is implementable in Nash equilibrium.

Claim (a) in Corollary 1 implies the main implementation results by Kara and Sönmez (1996), and Sönmez (1996) in the case of marriage markets. For housing markets, $\widetilde{\mathcal{R}}$ does not include all profiles with strict preferences over objects and without externalities (see Example 7) so our result does not imply the results in Sönmez (1996) for this environment nor it is implied by his.

\subsection{The limits of implementation}

First, we prove that the whole $\alpha$-individually rational and efficient rule is implementable in Nash equilibrium on $\widetilde{\mathcal{P}}$, the domain of strict preference profiles in which the $\alpha$-core is nonempty. Similarly, the $\alpha$-individually rational and weakly efficient rule is implementable in Nash equilibrium on $\widetilde{\mathcal{R}}^{\prime}$, the domain of preference profiles in which the weak $\alpha$-core is nonempty.

Proposition 6 The following claims hold: 
(a) The $\alpha$-individually rational and efficient correspondence $\alpha \mathscr{I} \mathscr{E}: \widetilde{\mathcal{P}} \rightrightarrows \mathcal{A}^{f}$ is implementable in Nash equilibrium.

(b) The $\alpha$-individually rational and weakly efficient correspondence $\alpha \mathscr{I} \mathscr{W} \mathscr{E}: \widetilde{\mathcal{R}}^{\prime} \rightrightarrows \mathcal{A}^{f}$ is implementable in Nash equilibrium.

The proof of Proposition 6 is identical to the proof of Theorem 1 and thus omitted. ${ }^{15}$ The next example shows that claim $(a)$ does not hold if the domain is $\widetilde{\mathcal{R}}$. The reason is that a monotonic transformation of preferences can transform an efficient allocation into a one which weakly efficient but not efficient, if the domain includes non strict preference profiles.

Example 3 Let $\alpha \mathscr{I} \mathscr{E}: \widetilde{\mathcal{R}} \rightrightarrows \mathcal{A}^{f}$. Let $N=\{1,2,3\}$. The initial endowment is $e=$ $\left(e_{1}, e_{2}, e_{3}\right)$. Let $a=\left(e_{1}, e_{3}, e_{2}\right)$. Let $\mathcal{A}^{f}$ be the set of all allocations. Consider the following strict preference profile:

$$
\begin{aligned}
& R_{1}: e P_{1} a P_{1} x, \quad \forall x \in \mathcal{A}^{f} \backslash\{a, e\} ; \\
& R_{2}: a P_{2} e P_{2} x \quad \forall x \in \mathcal{A}^{f} \backslash\{a, e\} ; \\
& R_{3}: a P_{3} e P_{3} x \quad \forall x \in \mathcal{A}^{f} \backslash\{a, e\} .
\end{aligned}
$$

We have $\alpha \mathscr{C}(R)=\mathscr{W} \alpha \mathscr{C}(R)=\{a\} .{ }^{16}$ Moreover $\alpha \mathscr{I} \mathscr{E}(R)=\{a, e\}$. We now modify the preferences of agent 1 into $R_{1}^{\prime}: e I_{1}^{\prime} a P_{1}^{\prime} x, \forall x \in \mathcal{A}^{f} \backslash\{a, e\}$. Let $R_{i}^{\prime}=R_{i}$ for $i \in\{2,3\}$. Notice that $\alpha \mathscr{C}\left(R^{\prime}\right)=\mathscr{W} \alpha \mathscr{C}\left(R^{\prime}\right)=\{a\}$, thus $R^{\prime} \in \widetilde{\mathcal{R}}$. We have $L\left(e, R_{i}\right)=L\left(e, R_{i}^{\prime}\right)$ for all $i \in N$. However $e \notin \alpha \mathscr{I} \mathscr{E}\left(R^{\prime}\right)=\{a\}$. It follows that $\alpha \mathscr{I} \mathscr{E}$ is not monotonic if the domain is $\widetilde{\mathcal{R}}$.

In Example 3, allocation $e$ is efficient under $R$ but does not belong to $\alpha \mathscr{C}(R)$. Transforming $R$ into $R^{\prime}$, the lower contour set of $e$ is unaffected, however $e$ is no longer efficient under $R^{\prime}$. Thus, any monotonic $S C R, \Omega$ implementing $e$ under $R$ should implement $e$ under $R^{\prime}$. In particular $\Omega$ would not be efficient. The example does not apply to strict

\footnotetext{
${ }^{15}$ More in general, claim $(b)$ holds for the domain in which correspondence $\alpha \mathscr{I} \mathscr{W} \mathscr{E}$ is nonempty valued.

${ }^{16}$ Coalition $\{2,3\}$ strongly $\alpha$-blocks all allocations except $a$.
} 
preferences profiles, where no allocation is weakly efficient.

Next, we generalize the intuition of Example 3 and prove that the $\alpha$-core correspondence is the maximal monotonic, $\alpha$-individually rational, and efficient $S C R$ having $\widetilde{\mathcal{R}}$ as domain.

Theorem 2 Let $\Omega$ be a monotonic, $\alpha$-individually rational, and efficient $S C R, \Omega: \mathcal{D} \rightrightarrows$ $\mathcal{A}^{f}$ such that $\widetilde{\mathcal{R}} \subseteq \mathcal{D}$. Then $\Omega(R) \subseteq \alpha \mathscr{C}(R)$ for all $R \in \widetilde{\mathcal{R}}$.

Proof. We prove by contradiction that $\Omega(R) \subseteq \alpha \mathscr{C}(R)$ for all $R \in \widetilde{\mathcal{R}}$. Assume that there exists $R \in \widetilde{\mathcal{R}}$ such that $\Omega(R) \backslash \alpha \mathscr{C}(R) \neq \emptyset$. Let $a \in \Omega(R) \backslash \alpha \mathscr{C}(R)$. Let $b \in \alpha \mathscr{C}(R)=\mathscr{W} \alpha \mathscr{C}(R)$. Let $N_{1}(R)=\left\{i \in N \mid b R_{i} a\right\}$ and $N_{2}(R)=\left\{i \in N \mid a P_{i} b\right\}$. We have $N_{1}(R) \cup N_{2}(R)=N$ and $N_{1}(R) \cap N_{2}(R)=\emptyset$. Moreover $N_{1}(R) \neq \emptyset$ and $N_{2}(R) \neq \emptyset{ }^{17}$ Since $b$ is efficient, there exists $i \in N_{1}(R)$ such that $b P_{i} a$. Let $\widehat{R}$ be an arbitrary strict preference relation over $\mathcal{A}^{f}$ such that $b \widehat{P} c$ for all $c \in \mathcal{A}^{f} \backslash\{b\}$. We now introduce an auxiliary preference profile, $R^{\prime}$ such that for all $i \in N_{1}(R)$ :

(i) $c P_{i}^{\prime} a P_{i}^{\prime} d$ for all $c \in \mathcal{A}^{f} \backslash L\left(a, R_{i}\right)$ and for all $d \in L\left(a, R_{i}\right) \backslash\{a, b\}$;

(ii) $b I_{i} a \Rightarrow b I_{i}^{\prime} a$

(iii) $c P_{i} d \Rightarrow c P_{i}^{\prime} d$ for all $c, d \in \mathcal{A}^{f}$

(iv) $\left[c I_{i} d\right.$ and $\left.c \widehat{P} d\right] \Rightarrow c P_{i}^{\prime} d$ for all $c, d \in \mathcal{A}^{f} \backslash\{a\}$

For all $i \in N_{2}(R)$ :

(v) $c P_{i}^{\prime} a I_{i}^{\prime} b P_{i}^{\prime} d$ for all $c \in \mathcal{A}^{f} \backslash L\left(a, R_{i}\right)$ and for all $d \in L\left(a, R_{i}\right) \backslash\{a, b\}$;

(vi) $c P_{i} d \Rightarrow c P_{i}^{\prime} d$ for all $c, d \in \mathcal{A}^{f} \backslash\{a, b\}$;

(vii) $\left[c I_{i} d\right.$ and $\left.c \widehat{P} d\right] \Rightarrow c P_{i}^{\prime} d$ for all $c, d \in \mathcal{A}^{f} \backslash\{a, b\}$.

${ }^{17}$ If $N_{1}(R)=\emptyset$ then $a P_{i} b$ for all $i \in N$ and $N$ strongly $\alpha$-blocks $b$ which contradicts $b \in \mathscr{W} \alpha \mathscr{C}(R)$. Similarly, if $N_{2}(R)=\emptyset$ two cases could occur: (1) $a I_{i} b$ for all $i \in N$, then $b \in \alpha \mathscr{C}(R) \Rightarrow a \in \alpha \mathscr{C}(R)$ which yields a contradiction; (2) $b R_{i} a$ for all $i \in N$ and $b P_{i} a$ for some $i \in N$, then $a \notin \mathscr{E}(R)$ which yields a contradiction. 
First, we prove that $R^{\prime} \in \widetilde{\mathcal{R}}$. We begin proving that $\alpha \mathscr{C}\left(R^{\prime}\right) \neq \emptyset$. It is enough to prove that $b \in \alpha \mathscr{C}\left(R^{\prime}\right)$. We prove the claim by contradiction. Assume $b \notin \alpha \mathscr{C}\left(R^{\prime}\right)$. Then, there exists a coalition $T$ which $\alpha$-blocks $b$ under $R^{\prime}$. From $(i i i),(i v),(v)$ in the definition of $R^{\prime}$ and the definition of $\widehat{R}$, coalition $T \alpha$-blocks $b$ under $R$ as well, which yields a contradiction.

Now, we prove $\alpha \mathscr{C}\left(R^{\prime}\right)=\mathscr{W} \alpha \mathscr{C}\left(R^{\prime}\right)$. It suffices to prove $\mathscr{W} \alpha \mathscr{C}\left(R^{\prime}\right) \subseteq \alpha \mathscr{C}\left(R^{\prime}\right)$. We prove this claim by contradiction. Assume that there exists $c \in \mathcal{A}^{f}$ such that $c \in \mathscr{W} \alpha \mathscr{C}\left(R^{\prime}\right)$ but $c \notin \alpha \mathscr{C}\left(R^{\prime}\right)$. Notice that $c \in\{a, b\}$, because, from (iv) and (vii) in the definition of $R^{\prime}$, there exist no $d \notin\{a, b\}$ and $f \in \mathcal{A}^{f}$ such that $d I_{i} f$. Moreover, $c \neq b$ because $b \in \alpha \mathscr{C}\left(R^{\prime}\right)$. Then, $c=a$. Since $a \notin \alpha \mathscr{C}(R)=\mathscr{W} \alpha \mathscr{C}(R)$, there exists $T$ which strongly $\alpha$-blocks $a$ under $R$. From $(i)$ and $(v)$ in the definition of $R^{\prime}$, the coalition $T$ strongly $\alpha$-blocks $a$ under $R^{\prime}$ as well. Then $a \notin \mathscr{W} \alpha \mathscr{C}\left(R^{\prime}\right)$, which yields a contradiction.

We have $L\left(a, R_{i}\right)=L\left(a, R_{i}^{\prime}\right)$ for all $i \in N$. From $(i i),(i i i)$, and $(v)$ in the definition of $R^{\prime}, b R_{i}^{\prime} a$ for all $i \in N$. From (iii) and the efficiency of $b$, there exists $i \in N_{1}(R)$ such that $b P_{i}^{\prime} a$. Then $a$ is not efficient under $R^{\prime}$ which implies $a \notin \Omega\left(R^{\prime}\right)$ which contradicts the monotonicity of $\Omega$.

Then, we approach the issue of minimality. In Proposition 3, we prove that the core correspondence (as well as the $\alpha$-core correspondence) is not implementable, when the preference domain is $\mathcal{R}^{|N|}$, thus we restrict our attention to $\widetilde{\mathcal{R}}$. By definition $\mathscr{C}(R) \subseteq$ $\alpha \mathscr{C}(R)$ for all $R \in \widetilde{\mathcal{R}}$. Thus we start by considering the core correspondence. Since the core may be empty in $\widetilde{\mathcal{R}}$, we restrict the domain to $\widetilde{\mathcal{R}}_{\mathscr{C}}=\{R \in \widetilde{\mathcal{R}} \mid \mathscr{C}(R) \neq \emptyset\}$. Example 4 shows that the core correspondence is not implementable on $\widetilde{\mathcal{R}}_{\mathscr{C}}$.

Example 4 Let $N=\{1,2,3,4\}$. The initial endowment is $e=\left(e_{1}, e_{2}, e_{3}, e_{4}\right)$. Let $a=$ $\left(e_{2}, e_{1}, e_{3}, e_{4}\right), b=\left(e_{2}, e_{1}, e_{4}, e_{3}\right), c=\left(e_{3}, e_{4}, e_{2}, e_{1}\right)$. Let $\mathcal{A}^{f}$ be the set of all allocations. Consider the following preferences:

$$
\begin{aligned}
& R_{1}: a P_{1} c P_{1} b P_{1} e I_{1} x P_{1} y, \quad \forall x \in A_{1} \backslash\{e\} \text { and } \forall y \in \mathcal{A}^{f} \backslash\left(\{a, b, c\} \cup A_{1}\right) ; \\
& R_{2}: c P_{2} a P_{2} b P_{2} e I_{2} x P_{2} y, \quad \forall x \in A_{2} \backslash\{e\} \text { and } \forall y \in \mathcal{A}^{f} \backslash\left(\{a, b, c\} \cup A_{2}\right) ;
\end{aligned}
$$




$$
\begin{aligned}
& R_{3}: c P_{3} a I_{3} e I_{3} x P_{3} y, \quad \forall x \in A_{3} \backslash\{a, e\} \text { and } \forall y \in \mathcal{A}^{f} \backslash\left(\{c\} \cup A_{3}\right) \\
& R_{4}: c P_{4} a I_{4} e I_{4} x P_{4} y, \quad \forall x \in A_{4} \backslash\{a, e\} \text { and } \forall y \in \mathcal{A}^{f} \backslash\left(\{c\} \cup A_{4}\right) .
\end{aligned}
$$

We have $\mathscr{C}(R)=\{a, c\}$. Let $R_{2}^{\prime}: c I_{2}^{\prime} a P_{2}^{\prime} b P_{2}^{\prime} e I_{2} x P_{2} y, \quad \forall x \in A_{2} \backslash\{e\}$ and $y \in \mathcal{A}^{f} \backslash$ $\left(\{a, b, c\} \cup A_{2}\right)$ and let $R^{\prime}=\left(R_{1}, R_{2}^{\prime}, R_{3}, R_{4}\right)$. Since $\alpha \mathscr{C}(R)=\mathscr{W} \alpha \mathscr{C}(R)=\alpha \mathscr{C}\left(R^{\prime}\right)=$ $\mathscr{W} \alpha \mathscr{C}\left(R^{\prime}\right)=\{a, c\}, R, R^{\prime} \in \widetilde{\mathcal{R}}$. We have $L\left(c, R_{i}\right) \subseteq L\left(c, R_{i}^{\prime}\right)$ for all $i \in N$, however $c \notin \mathscr{C}\left(R^{\prime}\right)$ then the core correspondence is not monotonic. Notice that $\mathscr{C}\left(R^{\prime}\right)=\{a\} \neq \emptyset$.

We next introduce a restriction on the set of feasible allocations.

Assumption 1 If $a \in \mathcal{A}^{f}$ and $a(i)=e_{i}$ for some $i \in N$, then $a=e$.

If Assumption 1 holds, the only allocation in which an agent keeps her endowment is $e$. In other words, the assumption requires that if an individual does not participate to the exchange, nobody will participate. Under this assumption, individual rationality and $\alpha$-individual rationality are equivalent.

We next prove that under Assumption 1 the $\alpha$-core correspondence is the minimal monotonic, $\alpha$-individually rational, and efficient correspondence defined on $\widetilde{\mathcal{R}}$.

Theorem 3 Let Assumption 1 be satisfied. Let $\Omega$ be a monotonic, $\alpha$-individually rational, and efficient $S C R, \Omega: \mathcal{D} \rightrightarrows \mathcal{A}^{f}$ such that $\widetilde{\mathcal{R}} \subseteq \mathcal{D}$. Then $\alpha \mathscr{C}(R) \subseteq \Omega(R)$, for all $R \in \widetilde{\mathcal{R}}$.

Proof. Let $R \in \widetilde{\mathcal{R}}$ and let $a \in \alpha \mathscr{C}(R)$. We will prove that $a \in \Omega(R)$. Since $a \in \alpha \mathscr{I}(R)$, Assumption 1 implies $a R_{i} e$ for all $i \in N$. We start introducing an auxiliary preference profile, $R^{\prime}$, defined as follows, for all $i \in N$ :

(i) $b R_{i}^{\prime} c \Leftrightarrow b R_{i} c$ for all $b, c \in \mathcal{A}^{f} \backslash(\{e, a\})$;

(ii) $e P_{i}^{\prime} b$ for all $b \in L\left(a, R_{i}\right) \backslash(\{e, a\})$;

(iii) $b P_{i}^{\prime} a P_{i}^{\prime} c$ for all $b \in \mathcal{A}^{f} \backslash L\left(a, R_{i}\right)$ and for all $c \in L\left(a, R_{i}\right) \backslash\{a\}$.

We now prove $R^{\prime} \in \widetilde{\mathcal{R}}$. Since $a \in \alpha \mathscr{C}(R)$, the definition of $R^{\prime}$ implies $a \in \alpha \mathscr{C}\left(R^{\prime}\right)$ which implies that $\alpha \mathscr{C}\left(R^{\prime}\right) \neq \emptyset$. Next, we prove that $\alpha \mathscr{C}\left(R^{\prime}\right)=\mathscr{W} \alpha \mathscr{C}\left(R^{\prime}\right)$, which implies 
that $R^{\prime} \in \widetilde{\mathcal{R}}$. We always have $\alpha \mathscr{C}\left(R^{\prime}\right) \subseteq \mathscr{W} \alpha \mathscr{C}\left(R^{\prime}\right)$. We thus prove the other inclusion, $\mathscr{W} \alpha \mathscr{C}\left(R^{\prime}\right) \subseteq \alpha \mathscr{C}\left(R^{\prime}\right)$. Since $a$ is $\alpha$-individually rational, by (iii) and Assumption 1, if $e \neq a, e$ is $\alpha$-blocked and strongly $\alpha$-blocked by the coalition $T=N$. Let $i \in N$. By (ii), each $b \in L\left(a, R_{i}^{\prime}\right) \backslash(\{e, a\})$ is $\alpha$-blocked and strongly $\alpha$-blocked by agent $i$. Thus, if $b \in \mathscr{W} \alpha \mathscr{C}\left(R^{\prime}\right)$, and $b \neq a$, then $b P_{i}^{\prime} a$ for all $i \in N$ and $b \neq e$. In particular, $a \notin \mathscr{E}\left(R^{\prime}\right)$, which contradicts $a \in \alpha \mathscr{C}\left(R^{\prime}\right)$. We conclude that $\{a\}=\mathscr{W} \alpha \mathscr{C}\left(R^{\prime}\right)=\alpha \mathscr{C}\left(R^{\prime}\right)$, thus $R^{\prime} \in \widetilde{\mathcal{R}}$

We next prove that $a$ is the only efficient and $\alpha$-individually rational allocation under $R^{\prime}$. Notice that $L\left(a, R_{i}\right)=L\left(a, R_{i}^{\prime}\right)$ for every $i \in N$. Since $L\left(a, R_{i}\right) \subseteq L\left(a, R_{i}^{\prime}\right)$ for every $i \in N$ and because $\alpha \mathscr{C}$ is monotonic in $\widetilde{\mathcal{R}}, a \in \alpha \mathscr{C}\left(R^{\prime}\right) \subseteq \mathscr{E}\left(R^{\prime}\right) \cap \alpha \mathscr{I}\left(R^{\prime}\right)$. We prove by contradiction that $\mathscr{E}\left(R^{\prime}\right) \cap \alpha \mathscr{I}\left(R^{\prime}\right)=\{a\}$. Assume there exists $b \in \mathscr{E}\left(R^{\prime}\right) \cap \alpha \mathscr{I}\left(R^{\prime}\right) \backslash\{a\}$. Since $a \in \mathscr{E}\left(R^{\prime}\right),($ iii $)$ in the definition of $R^{\prime}$ implies that there exists $i \in N$ such that $a P_{i}^{\prime} b$. Since $a \in \alpha \mathscr{I}\left(R^{\prime}\right),(i i)$ in the definition of $R^{\prime}$ and Assumption 1 imply that $e P_{i}^{\prime} b$, then $b$ is $\alpha$-blocked by $i$, which contradicts $b \in \alpha \mathscr{I}\left(R^{\prime}\right)$. Therefore, $\mathscr{E}\left(R^{\prime}\right) \cap \alpha \mathscr{I}\left(R^{\prime}\right)=\{a\}$ thus $\Omega\left(R^{\prime}\right)=\{a\}$. Then $a \in \Omega(R)$ because $L\left(a, R_{i}^{\prime}\right) \subseteq L\left(a, R_{i}\right)$ for every $i \in N$ and $\Omega$ is monotonic.

Theorem 3 above and Theorem 1 in Sönmez (1996) do not imply that the core and the $\alpha$-core coincide on $\widetilde{\mathcal{R}}$ whenever the core exists in $\widetilde{\mathcal{R}}$. Indeed, in Example $4, \mathscr{C}\left(R^{\prime}\right) \subsetneq$ $\alpha \mathscr{C}\left(R^{\prime}\right)$.

From Theorem 2 and Theorem 3, it follows that, under Assumption 1, the $\alpha$-core is the unique monotonic (and thus implementable), $\alpha$-individually rational, and efficient social choice rule having $\widetilde{\mathcal{R}}$ as domain.

Corollary 2 Let Assumption 1 be satisfied. Let $\Omega$ be a monotonic, $\alpha$-individually rational, and efficient $S C R, \Omega: \mathcal{D} \rightrightarrows \mathcal{A}^{f}$ such that $\widetilde{\mathcal{R}} \subseteq \mathcal{D}$. Then $\Omega(R)=\alpha \mathscr{C}(R)$, for all $R \in \widetilde{\mathcal{R}}$.

Enlarging the preference domain, in general, forces to implement "larger" correspondences. An example is the case in which the preference domain is $\widetilde{\mathcal{R}}^{\prime}$. In this case, monotonicity forces to implement the whole weak $\alpha$-core. On the contrary, restricting the domain to strict preferences does not relax the result of Theorem 3. 
Proposition 7 Let Assumption 1 be satisfied. Let $\Omega$ be a monotonic and $\alpha$-individually rational $S C R, \Omega: \mathcal{D} \rightrightarrows \mathcal{A}^{f}$.

(a) If $\widetilde{\mathcal{R}}^{\prime} \subseteq \mathcal{D}$ and $\Omega$ is weakly efficient, then $\mathscr{W} \alpha \mathscr{C}(R) \subseteq \Omega(R)$, for all $R \in \widetilde{\mathcal{R}}^{\prime}$

(b) If $\widetilde{\mathcal{P}} \subseteq \mathcal{D}$ and $\Omega$ is efficient, then $\alpha \mathscr{C}(R) \subseteq \Omega(R)$, for all $R \in \widetilde{\mathcal{P}}$.

The proof of both results is identical to the proof of Theorem 3 and thus omitted.

Claim (a) implies that it is impossible to implement an $\alpha$-individually rational and efficient $S C R$ if the domain is $\widetilde{\mathcal{R}}^{\prime}$ or larger. In this case, the designer has to settle on weakly efficient $S C R$. Claim (b) implies that the $\alpha$-core is the minimal implementable $S C R$ for marriage markets, roommate problems, and housing markets with externality, strict preferences, and nonempty $\alpha$-cores. Future research should establish whether restricting the domain in $(a)$ or in $(b)$ helps relaxing the results.

We do not know whether Assumption 1 is necessary for Theorem 3 to hold. However, the next example shows that claim $(b)$ of Proposition 7 does not hold without Assumption 1.

Example 5 Let $R \in \widetilde{\mathcal{P}}$ and $H(R)=\left\{a \in \mathcal{A}^{f} \mid a R_{i} e \forall i \in N\right.$ and $a P_{j}$ e for some $\left.j \in N\right\}$. Let $\Omega^{*}: \widetilde{\mathcal{P}} \rightrightarrows \mathcal{A}^{f}$ such that

$$
\Omega^{*}(R)=\left\{\begin{array}{l}
\{e\} \text { if } e \in \mathscr{E}(R) ; \\
H(R) \cap \mathscr{E}(R) \text { if } e \notin \mathscr{E}(R) .
\end{array}\right.
$$

Social choice rule $\Omega^{*}$ is nonempty, monotonic, $\alpha$-individually rational, and efficient. Let $N=\{1,2,3\}$. The initial endowment is $e=\left(e_{1}, e_{2}, e_{3}\right)$. Let $a=\left(e_{1}, e_{3}, e_{2}\right)$. Let $\mathcal{A}^{f}$ be the set of all allocations. Consider the following strict preference profile:

$$
\begin{aligned}
& R_{1}: e P_{1} a P_{1} x, \quad \forall x \in \mathcal{A}^{f} \backslash\{a, e\} ; \\
& R_{2}: a P_{2} e P_{2} x \quad \forall x \in \mathcal{A}^{f} \backslash\{a, e\} ; \\
& R_{3}: a P_{3} e P_{3} x \quad \forall x \in \mathcal{A}^{f} \backslash\{a, e\} .
\end{aligned}
$$


Since $a \in \mathcal{A}^{f}$, Assumption 1 is not satisfied. We have $\alpha \mathscr{C}(R)=\mathscr{W} \alpha \mathscr{C}(R)=\{a\}$. However $\Omega^{*}(R)=\{e\}$.

Social choice rule $\Omega^{*}$ can be extended to $\widetilde{\mathcal{R}}$ but it is not monotonic on $\widetilde{\mathcal{R}}$. Let $R_{1}^{\prime}$ : $e I_{1}^{\prime} a P_{1}^{\prime} x, \forall x \in \mathcal{A}^{f} \backslash\{a, e\}$. Let $R_{i}^{\prime}=R_{i}$ for $i \in\{2,3\}$. Notice that $\alpha \mathscr{C}\left(R^{\prime}\right)=\mathscr{W} \alpha \mathscr{C}\left(R^{\prime}\right)=$ $\{a\}$, thus $R^{\prime} \in \widetilde{\mathcal{R}}$. We have $L\left(e, R_{i}\right)=L\left(e, R_{i}^{\prime}\right)$ for all $i \in N$. However $e \notin \Omega^{*}\left(R^{\prime}\right)=\{a\}$. It follows that $\Omega^{*}$ is not monotonic on $\widetilde{\mathcal{R}}$.

\section{Conclusions}

We have proved that no $\alpha$-individually rational and efficient mechanism is implementable in dominant strategies in allocation problems with externalities, when the set of admissible preferences of each agent includes all strict preferences. In particular, every strategy-proof and efficient mechanism does not provide all agents the right incentives to participate: for some preference profile, one agent will be strictly better off by not participating to the mechanism, independently on the behavior of the other agents. The $\alpha$-core is implementable in Nash equilibrium in environments in which the $\alpha$-core equals the weak $\alpha$-core and both are nonempty. In this domain the $\alpha$-core is the maximal implementable $\alpha$-individually rational and efficient social choice rule. It is also the unique implementable solution which is $\alpha$-individually rational and efficient under an additional mild restriction on the set of feasible allocations.

In front of a general impossibility result, we have relaxed the equilibrium concept and studied the implementation of social choice rules in Nash equilibrium. Employing a complementary approach, future research, should establish the maximal preference domain on which there is a strategy-proof, $\alpha$-individually rational, and efficient social choice function.

\section{References}

[1] Aumann, R.J., 1961. The core of a cooperative game without side payments. T. Am. Math. Soc. 98, 539-552. https://doi.org/10.1090/S0002-9947-1961-0127437-2. 
[2] Aumann, R.J., and Peleg, B., 1960. Von Neumann-Morgenstern solutions to cooperative games without side payments. Bull. Am. Math. Soc. 66, 173-179. https://doi.org/10.1090/S0002-9904-1960-10418-1.

[3] Basile, A., Scalzo, V. 2020. Non-emptiness of the alpha-core: sufficient and necessary conditions. Int. J. Game Theory 49, 1143-1153. https://doi.org/10.1007/s00182-02000736-y.

[4] Barberà, S., and Dutta, B. 1982. Implementability via protective equilibria. J. Math. Econ. 10, 49-65. https://doi.org/10.1016/0304-4068(82)90005-2.

[5] Barberà, S., and Dutta, B. 1995. Protective behavior in matching models. Games Econ. Behav. 8, 281-296. https://doi.org/10.1016/S0899-8256(05)80002-8.

[6] Chung, K.S., 2000. On the Existence of Stable Roommate Matchings. Games Econ. Behav. 33, 206-230. https://doi.org/10.1006/game.1999.0779.

[7] Contreras, J.L., Torres-Martínez, J.P. 2021. The roommate problem with externalities. Int. J. Game Theory 50, 149-165. https://doi.org/10.1007/s00182-020-00743-z.

[8] Ehlers, L., 2018. Strategy-proofness and essentially single-valued cores revisited. J. Econ. Theory 176, 393-407. https://doi.org/10.1016/j.jet.2018.04.008.

[9] Fonseca-Mairena, M.H., Triossi, M., 2019. Incentives and implementation in marriage markets with externalities. Econ. Lett. 185. https://doi.org/10.1016/j.econlet.2019.108688.

[10] Gale, D., Shapley, L. S., 1962. College Admissions and the Stability of Marriage. T. Am. Math. Mon. 69, 9-15. https://doi.org/10.1080/00029890.1962.11989827.

[11] Gibbard, A., 1973. Manipulation of voting schemes: a general result. Econometrica 41, 587-601. https://doi.org/10.2307/1914083.

[12] Hong, M., Park, J., 2018. Core and Top Trading Cycles in a Market with Indivisible Goods and Externalities. Mimeo, https://ssrn.com/abstract=3710055. last accessed 05/07/2021. 
[13] Kajii, A., 1992. A generalization of Scarf's theorem: $\alpha$-core existence theorem without transitivity or completeness, J. Econ. Theory 56, 194-205. https://doi.org/10.1016/0022-0531(92)90076-T.

[14] Kara, T., Sönmez, T., 1996. Nash implementation of matching rules. J. Econ. Theory 68, 425-439. https://doi.org/10.1006/jeth.1996.0024.

[15] Kara, T., Sönmez, T., 1997. Implementation of college admission rules. Econ. Theory 9, 197-218. https://doi.org/10.1007/BF01213799.

[16] Martins-da-Rocha, V.F., Yannelis, N.C., 2011. Non-emptiness of the alpha-core. Mimeo, FGV EPGE Economics Working Papers 716, http://bibliotecadigital.fgv.br/dspace/bitstream/10438/8024/5/Non-emptinessof-the-alpha-core.pdf, last accessed 05/07/2021.

[17] Maskin, E., 1999. Nash equilibrium and welfare optimality. Rev. Econ. Stud. 66, 23-38. https://doi.org/10.1111/1467-937X.00076.

[18] Mumcu, A., Saglam, I., 2007. The core of a housing market with externalities. Econ. Bulletin 55, 1-5. http://www.accessecon.com/pubs/EB/2007/Volume3/EB07C70026A.pdf

[19] Mumcu, A., Saglam, I., 2010. Stable one-to-one matchings with externalities. Math. Soc. Sci., 60(2), 154-159. https://doi.org/10.1016/j.mathsocsci.2010.05.002

[20] Prather, K.A., Wang, C.C., Schooley, R.T., 2020. Reducing transmission of SARSCoV-2. Science 368, 1422-1424, https://doi.org/10.1126/science.abc6197.

[21] Roth, A.E., Postlewaite, A., 1977. Weak versus strong domination in a market with indivisible goods. J. Math. Econ. 4, 131-137. https://doi.org/10.1016/03044068(77)90004-0.

[22] Sasaki, H., Toda, M., 1996. Two-sided matching problems with externalities. J. Econ. Theory 70, 93-108. https://doi.org/10.1006/jeth.1996.0077. 
[23] Satterthwaite, M.A., 1975. Strategy-proofness and Arrow's conditions: Existence and correspondence theorems for voting procedures and social welfare functions. J. of Econ. Theory 10, 187-217. https://doi.org/10.1016/0022-0531(75)90050-2.

[24] Shapley, L., Scarf, H., 1974. On cores and indivisibility. J. Math. Econ. 1, 23-37. https://doi.org/10.1016/0304-4068(74)90033-0.

[25] Sönmez, T., 1996. Implementation in Generalized Matching Problems. J. Math. Econ. 26, 429-439. https://doi.org/10.1016/S0304-4068(96)00758-6

[26] Sönmez, T., 1999. Strategy-proofness and essentially single-valued cores. Econometrica 67, 677-689. https://doi.org/10.1111/1468-0262.00044.

[27] Yamato, T., 1992. On Nash implementation of social choice correspondences. Games Econ. Behav. 4, 484-492. https://doi.org/10.1016/0899-8256(92)90051-S.

\section{Appendix}

Let $\mathcal{R}^{\text {wes }}$ be the domain of profiles with strict preferences over objects and without externalities as a subset of $\mathcal{R}^{|N|}$, which is $\mathcal{R}^{\text {wes }}=\left\{R \in \mathcal{R}^{|N|} \mid a I_{i} b \Leftrightarrow a(i)=b(i), \forall i \in N\right\}$. Let $\mathcal{R}^{\text {seg }}$ be the domain of strict egocentric preference profiles. Profile $R=\left(R_{i}\right)_{i \in N} \in \mathcal{R}^{\text {seg }}$ if and only if it satisfies: ( $i$ ) for all $a, b \in \mathcal{A}^{f}, a I_{i} b \Rightarrow a=b ;(i i)$ for all $a, b \in \mathcal{A}^{f}$ such that $a(i) \neq b(i), a P_{i} b \Leftrightarrow(a(i), c(-i)) P_{i}(b(i), d(-i))$ for all $c(-i), d(-i)$ such that $(a(i), c(-i)),(b(i), d(-i)) \in \mathcal{A}^{f}$. Each profile in $\mathcal{R}^{\text {seg }}$ is strict, thus $\alpha \mathscr{C}(R)=\mathscr{W} \alpha \mathscr{C}(R)$ for all $R \in \mathcal{R}^{\text {seg }}$.

We associate a profile with strict preferences over objects and without externalities to each strict egocentric preference profile. Let $R \in \mathcal{R}^{\text {seg }}$, and define $\widehat{R}=\left(\widehat{R}_{i}\right)_{i \in N}$ as follows: for all $a, b \in \mathcal{A}^{f}$ and $i \in N:(i) a \widehat{I}_{i} b \Leftrightarrow a(i)=b(i) ;(i i) a \widehat{P}_{i} b \Leftrightarrow a(i) \neq b(i)$ and $a P_{i} b$. Since $R \in \mathcal{R}^{\text {seg }}, \widehat{R}$ is well defined and $\widehat{R} \in \mathcal{R}^{\text {wes }}$.

For roommate problems, let $\mathcal{R}^{\text {wesno }}$ be the set of profiles with strict preferences over objects and without externalities that satisfy the no odd rings condition (Chung, 2000) and let $\mathcal{R}^{\text {segno }}$ be the set of strict egocentric preference profiles, $R$ such that $\widehat{R} \in \mathcal{R}^{\text {wesno }}$. 
The core of an allocation problem with strict preferences over objects and without externalities is a subset of the $\alpha$-core of the associated strict egocentric preferences.

Lemma 3 For all $R \in \mathcal{R}^{\text {seg }}, \mathscr{C}(\widehat{R}) \subseteq \alpha \mathscr{C}(R)=\mathscr{W} \alpha \mathscr{C}(R)$

Proof. Let $R \in \mathcal{R}^{s e g}$ and consider the associated profile with strict preferences over objects and without externalities, $\widehat{R} \in \mathcal{R}^{\text {wes }}$. We prove the claim by contradiction. Let $a \in \mathscr{C}(\widehat{R})$ and assume that there exists $T \subseteq N$ which $\alpha$-blocks (and strongly $\alpha$-blocks) $a$ under $R$. Then, there exists $b \in \mathcal{A}^{f}$ with $\bigcup_{i \in T} b(i)=\bigcup_{i \in T} e_{i}$ such that, for all $i \in T, c P_{i} a$ for all $c \in \mathcal{A}^{f}$ such that $c(i)=b(i)$ for all $i \in T$. Thus, the set $T^{\prime}=\{j \in T \mid b(j) \neq a(j)\}$ is nonempty. Then, for all $i \in T, b \widehat{R}_{i} a$ and there exists $j \in T^{\prime} \subseteq T$ such that $b \widehat{P}_{j} a$. Then, $T$ blocks $a$ when preferences are $\widehat{R}$, which yields a contradiction.

The inclusion in Lemma 3, is, in general, strict. Indeed, in Example 2, $\mathscr{C}(\widehat{R})=\{a\}$ while $\alpha \mathscr{C}(R)=\mathscr{W} \alpha \mathscr{C}(R)=\{a, c\}$. Also, under strict egocentric preferences the core can be empty even if the core under the associated profile with strict preferences over objects and without externalities is nonempty as shown in Example 6.

Example 6 Let $N=\{1,2,3,4\}$. The initial endowment is $e=\left(e_{1}, e_{2}, e_{3}, e_{4}\right)$. Let $a=\left(e_{2}, e_{1}, e_{3}, e_{4}\right), b=\left(e_{2}, e_{1}, e_{4}, e_{3}\right), c=\left(e_{1}, e_{2}, e_{4}, e_{3}\right)$. Let $\mathcal{A}^{f}$ be the set of all allocations. Let $A_{i}\left(e_{j}\right)=\left\{a \in \mathcal{A}^{f} \mid a(i)=e_{j}\right\}$ be the set of allocations in which $i$ gets the $j$ 's endowment. Consider the following strict egocentric preferences:

$R_{1}: a P_{1} b P_{1} x P_{1} c P_{1} e P_{1} y P_{1} z, \forall x \in A_{1}\left(e_{2}\right) \backslash\{a, b\}, \forall y \in A_{1}\left(e_{1}\right) \backslash\{c, e\}, \forall z \in \mathcal{A}^{f} \backslash\left\{A_{1}\left(e_{1}\right) \cup A_{1}\left(e_{2}\right)\right\} ;$

$R_{2}: a P_{2} b P_{2} x P_{2} c P_{2} e P_{2} y P_{2} z, \quad \forall x \in A_{2}\left(e_{1}\right) \backslash\{a, b\}, \forall y \in A_{2}\left(e_{2}\right) \backslash\{c, e\}, \forall z \in \mathcal{A}^{f} \backslash\left\{A_{2}\left(e_{1}\right) \cup A_{2}\left(e_{2}\right)\right\} ;$

$R_{3}: c P_{3} b P_{3} x P_{3} a P_{3} e P_{3} y P_{3} z, \forall x \in A_{3}\left(e_{4}\right) \backslash\{c, b\}, \forall y \in A_{3}\left(e_{3}\right) \backslash\{a, e\}, \forall z \in \mathcal{A}^{f} \backslash\left\{A_{3}\left(e_{3}\right) \cup A_{3}\left(e_{4}\right)\right\} ;$

$R_{4}: c P_{4} b P_{4} x P_{4} a P_{4} e P_{4} y P_{4} z, \quad \forall x \in A_{4}\left(e_{3}\right) \backslash\{c, b\}, \forall y \in A_{4}\left(e_{4}\right) \backslash\{a, e\}, \forall z \in \mathcal{A}^{f} \backslash\left\{A_{4}\left(e_{3}\right) \cup A_{4}\left(e_{4}\right)\right\}$.

We have $\mathscr{C}(\widehat{R})=\alpha \mathscr{C}(R)=\mathscr{W} \alpha \mathscr{C}(R)=\{b\} .{ }^{18}$ However $\mathscr{C}(R)=\emptyset .^{19}$

\footnotetext{
${ }^{18}$ Coalition $\{3,4\}$ strongly $\alpha$-blocks $a$ and $\{1,2\}$ strongly $\alpha$-blocks $c$. The other allocations are strongly $\alpha$-blocked by the grand coalition with $b$.

${ }^{19}$ Allocation $b$ is blocked by $\{1,2\}$ with $a$.
} 
We now consider the relation between $\mathcal{R}^{\text {wes }}$ and $\widetilde{\mathcal{R}}$, and show that in marriage markets and roommate problems the $\alpha$-core and the weak $\alpha$-core coincide.

Lemma 4 Let $R \in \mathcal{R}^{\text {wes }}$. Then $\alpha \mathscr{C}(R)=\mathscr{W} \alpha \mathscr{C}(R)$ in marriage markets and roommate problems.

Proof. By contradiction, suppose that for some $R \in \mathcal{R}^{\text {wes }}$ there exists $a \in \mathscr{W} \alpha \mathscr{C}(R)$ such that $a \notin \alpha \mathscr{C}(R)$. Let $T$ be a blocking coalition, ${ }^{20}$ and let $b$ such that $(i) \bigcup_{i \in T} b(i)=$ $\bigcup_{i \in T} e_{i}$, $(i i) c R_{i} a$ for each $i \in T$ and for all $c \in \mathcal{A}^{f}$ such that $c(i)=b(i) \forall i \in T$, and (iii) there exists $j \in T$ such that $c P_{j} a$ for all $c \in \mathcal{A}^{f}$ such that $c(i)=b(i) \forall i \in T$. Since $a \in \mathscr{W} \alpha \mathscr{C}(R)$, coalition $\widetilde{T}=\left\{i \in T \mid c I_{i} a\right.$ for some $c \in \mathcal{A}^{f}$ such that $\left.c(i)=b(i) \forall i \in T\right\}$ is nonempty. Since $R \in \mathcal{R}^{\text {wes }}, c I_{i} a$ implies $c(i)=a(i)$. Let $j=c(i)$. Clearly, $j \in \widetilde{T}$. Consider the coalition $T \backslash \widetilde{T}$. We have $(i) \bigcup_{i \in T \backslash \widetilde{T}} b(i)=\bigcup_{i \in T \backslash \widetilde{T}} e_{i}$, and $(i i) c P_{i} a$ for each $i \in T \backslash \widetilde{T}$ and for all $c \in \mathcal{A}^{f}$ such that $c(i)=b(i) \forall i \in T \backslash \widetilde{T}$. Then the coalition $T \backslash \widetilde{T}$ strongly $\alpha$-blocks $a$ which yields a contradiction.

In housing markets, instead, the $\alpha$-core can be a strict subset of the weak $\alpha$-core as shown in Example 7.

Example 7 Let $N=\{1,2,3\}$. Let $\mathcal{A}^{f}=\{a, b, c, d, e, f\}$, where $a=\left(e_{1}, e_{3}, e_{2}\right), b=$ $\left(e_{2}, e_{1}, e_{3}\right), c=\left(e_{2}, e_{3}, e_{1}\right), d=\left(e_{3}, e_{1}, e_{2}\right), e=\left(e_{1}, e_{2}, e_{3}\right)$, and $f=\left(e_{3}, e_{2}, e_{1}\right)$. Consider the following profile with strict preferences over objects and without externalities:

$$
R_{1}: c I_{1} b P_{1} e I_{1} a P_{1} d I_{1} f ; \quad R_{2}: c I_{2} a P_{2} b I_{2} d P_{2} e I_{2} f ; \quad R_{3}: c I_{3} f P_{3} b I_{3} e P_{3} a I_{3} d .
$$

We have $\alpha \mathscr{C}(R)=\{c\}$ and $\mathscr{W} \alpha \mathscr{C}(R)=\{b, c\}{ }^{21}$

Proposition 4 (see also Section 4) integrates the previous findings and existence results from the literature about allocation problems without externalities.

\section{Proposition 4 The following claims hold:}

\footnotetext{
${ }^{20}$ Notice $|T|>1$ because if an agent $\alpha$-blocks allocation $a$, she strongly $\alpha$-blocks $a$.

${ }^{21}$ Agent 1 strongly $\alpha$-blocks $d$ and $f$. Coalition $N$ strongly $\alpha$-blocks $e$. Agent 3 strongly $\alpha$-blocks $a$. Coalition $N \alpha$-blocks $b$, but does not strongly $\alpha$-block $b$.
} 
(a) In marriage markets $\mathcal{R}^{\text {wes }} \cup \mathcal{R}^{\text {seg }} \subseteq \widetilde{\mathcal{R}}$.

(b) In housing markets $\mathcal{R}^{\text {seg }} \subseteq \widetilde{\mathcal{R}}$ and $\mathcal{R}^{\text {wes }} \cap \widetilde{\mathcal{R}} \neq \emptyset$.

(c) In roommate problems $\mathcal{R}^{\text {wesno }} \cup \mathcal{R}^{\text {segno }} \subseteq \widetilde{\mathcal{R}}$

\section{Proof.}

(a) From Lemma 3 and Gale and Shapley (1962), $\mathcal{R}^{\text {seg }} \subseteq \widetilde{\mathcal{R}}$. From Lemma 4 and Gale and Shapley (1962), $\mathcal{R}^{\text {wes }} \subseteq \widetilde{\mathcal{R}}$, which completes the proof of the claim.

(b) From Lemma 3 and Shapley and Scarf (1974), $\mathcal{R}^{\text {seg }} \subseteq \widetilde{\mathcal{R}}$. Next, we prove that $\mathcal{R}^{\text {wes }} \cap \widetilde{\mathcal{R}} \neq \emptyset$. Let $R \in \mathcal{R}^{\text {wes }}$ such that, for all $i \in N, e R_{i} b$ for all $b \in \mathcal{A}^{f}$. We prove that $R \in \widetilde{\mathcal{R}}$. For all $b \neq e$ there exists $j \in N$ such that $b \notin A_{j}$ and $c P_{j} b$ for all $c \in A_{j}$, then $j$ strongly $\alpha$-blocks $b$. We conclude that $\alpha \mathscr{C}(R)=\mathscr{W} \alpha \mathscr{C}(R)=\{e\}$ which completes the proof of the claim.

(c) From Lemma 3 and Chung (2000), $\mathcal{R}^{\text {segno }} \subseteq \widetilde{\mathcal{R}}$. From Lemma 4 and Chung (2000), $\mathcal{R}^{\text {wesno }} \subseteq \widetilde{\mathcal{R}}$, which completes the proof of the claim. 